\title{
Metabolic Disequilibrium and Aging: Modifying Favorably with Calorie Restriction and Calorie Restriction Mimetics
}

ISSN: 2578-0093

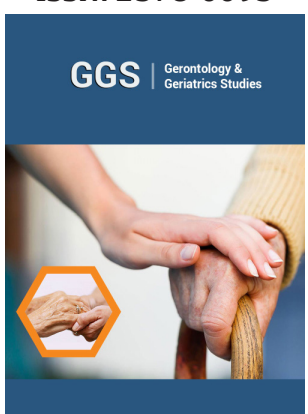

*Corresponding author: Vinod Nikhra, Senior Consultant and Faculty, Department of Medicine, New Delhi, India

Submission: 海 June 19, 2018

Published: 陋April 22, 2019

Volume 4 - Issue 5

How to cite this article: Vinod N. Metabolic Disequilibrium and Aging: Modifying Favorably with Calorie Restriction and Calorie Restriction Mimetics. Gerontol \& Geriatric stud.4(5). GGS.000596.2019. DOI: 10.31031/GGS.2019.04.000596

Copyright@ Vinod Nikhra, This article is distributed under the terms of the Creative Commons Attribution 4.0 International License, which permits unrestricted use and redistribution provided that the original author and source are credited.

\author{
Vinod Nikhra* \\ Department of Medicine, India
}

\begin{abstract}
Metabolic disease and aging: There are increasing evidence that metabolic disequilibrium and disorders influence the aging process and survival including the quality of life (QOL). The adipose tissue mediates various age-associated metabolic disorders such as insulin resistance (IR), metabolic syndrome (MetS), dyslipidemia and type 2 diabetes mellitus (T2DM), altogether which can negatively affect the lifespan and sends signals to modify the aging process in various tissues and organs. The role of master-switch and genomic guardian, p53 is important in this context.
\end{abstract}

Calorie restriction (Cr): The energy needs are determined by the body composition, especially the fat free lean mass and level of physical activity, and there is a change in nutritional needs during the middle age and later. The diet-gene interaction is a major determinant of health and illness, and the amount and type of food ingestion and caloric intake, in general, influence the health and life span. An excess calorie intake causes overnutrition and nutritional overload leading to increased adipose stores culminating as weight gain and obesity, which lead to IR, MetS, T2DM and other metabolic alterations. At the subcellular and cellular level there is increased potentially damaging exposure to reactive oxygen species (ROS).

Cr Mechanisms and pathways: There have been identified several metabolic and genetic pathways that govern food ingestion, metabolism and life span. Experimentally, the CR has been shown to achieve increased lifespan in a broad spectrum of life forms from yeast, nematode, fruit fly, rodents and primates, including Homo sapiens, endosing that a diet adequately fulfilling nutritional needs, but low in calories may improve health, prevent many late-onset diseases and extend the life span. But, the benefits of CR are not a passive result of lower caloric intake but the consequence of an active regulatory intervention mimicking the food scarcity and activating certain genetic and metabolic programs that result in various vital beneficial effects. The genetic and molecular studies in model organisms, in fact, suggest that CR is a regulated process, in which the SIRT (Silent Information Regulator 2) gene plays an important role.

Impact of Cr On Qol and disease: CR has long been recognized for its ability to extend life span and mitigate aging and stall disease processes in various organs. The data from animal and human studies indicate that the $\mathrm{CR}$ affects favorably several metabolic and molecular factors that modulate cardiovascular age-related alterations including cardiac and arterial stiffness, hypertension and heart rate variability. These effects, in combination with various other benefits, such as protection against adiposity, IR and T2DM, neurodegenerative diseases and cancer, suggest that the CR may have a major beneficial effect on health status, quality of life and life span in humans.

Keywords: Calorie restriction; Cardiovascular disease; Metabolic syndrome; Neuro-degenerative disorders p53; Reactive oxygen species; SIRT gene; Type 2 diabetes mellitus

\section{Metabolic Disequilibrium and Disorders}

There is increasing evidence that metabolic disequilibrium and disorders impact general health, aging process and survival including the quality of life (QOL). There is a changing pattern of diseases caused by overnutrition and unhealthy lifestyle. With weight gain and obesity, the adipose tissue mediates various metabolic disorders such as insulin resistance (IR), metabolic syndrome (MetS), dyslipidemia and type 2 diabetes mellitus (T2DM), altogether which negatively affect the lifespan and disfavorably modify the aging process in various tissues and organs through a number of metabolic pathways.

\section{Metabolic disorders and aging process}

As proved by various studies, the metabolic disorders are closely related with aging process. The development of IR is one of the most important risk factors for metabolic 
diseases in the middle aged and elderly. The decline in lean body mass and increase in body fat, particularly visceral adiposity, often accompany aging and contribute to the development of IR. The aging process correlates with $\beta$-cell functional decline, decreased $\beta$-cell proliferation capacity and enhanced sensitivity to apoptosis. The age-associated decline in mitochondrial function also contributes to IR. With aging, there occurs a progressive loss in mitochondrial function in various tissues, including skeletal muscle. Mitochondria are the major source of ROS generation and the alterations can lead to oxidative damage of macromolecules, including nuclear and mitochondrial DNA (mtDNA). Mitochondrial oxidative and phosphorylation functions may be reduced by about $40 \%$ in association with increased intramyocellular and intrahepatocellular lipid content and decreased insulin-stimulated glucose uptake [1]. The mitochondrial theory of aging posits that mitochondrial dysfunction may be a fundamental cause of cellular aging and senescence [2,3].

\section{Mets and viceral adiposity}

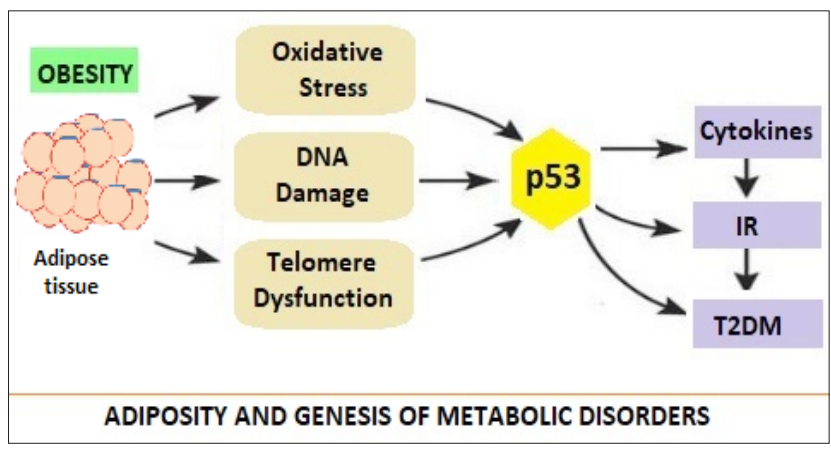

Figure 1: Adiposity, Stress Factors, p53 and Genesis of Metabolic Disorders [3].

The MetS is directly related to the accumulation of visceral adiposity in middle age associated with overnutrition and sedentary lifestyle. In addition, there may be a genetic predisposition to MetS. Apart from, insulin, two other hormones are associated with development of MetS, are the leptin and adiponectin which are produced by adipose tissue. The leptin levels are proportional to amount of adiposity and have anorectic effect and enhance metabolism. With obesity, there occurs resistance to leptin effects due to hypertriglyceridemia. Adiponectin enhances insulin sensitivity and decreases triglycerides levels. A low level of adiponectin accompanies the development of IR. The visceral fat (VF) is associated with increased cytokines such as interleukin-6 (IL-6) and tumor-necrosis factor $\alpha$ (TNF- $\alpha$ ), which play a major role in development of IR. The role of master-switch p53 is important in this context (Figure 1). Minamino et al proposed that the link between obesity, abnormal metabolism and Diabetes was p53. The activation of p53 in adipose tissue led to inflammation and insulin resistance culminating into T2DM [4]. In obese states, adipose tissue is subjected to oxidative stress, resulting DNA damage, telomere dysfunction and aging. This activates p53, which in turn causes production of pro-inflammatory cytokines, suppression of adiponectin, IR and progression to T2DM. In animal models, the inhibition of p53 activity in adipose tissue ameliorated the senescence-like changes, decreased the expression of proinflammatory cytokines and improved insulin resistance in mice with T2DM- like disease.

\section{The regulator role of p53}

The Role p53 is a 53-kdalton molecule, hence the name. It has been called the guardian of genome. It regulates the cell cycle, functions as tumor suppressor and plays an important role in apoptosis, genomic stability, and inhibition of angiogenesis. In normal cell p53 is in inactive form by its binding with mdm2. The various stressors lead to dissociation of the p53 and mdm2 complex (Figure 2). The p53 activation in adipose tissue appears to be a pro-aging signal, with negative influence on longevity. The p53 regulates lipid metabolism by binding to G6PD and inhibiting pentose phosphate pathway. In addition, p53 enhances fatty acid oxidation and modulates lipid transport by inducing the expression of multiple genes.

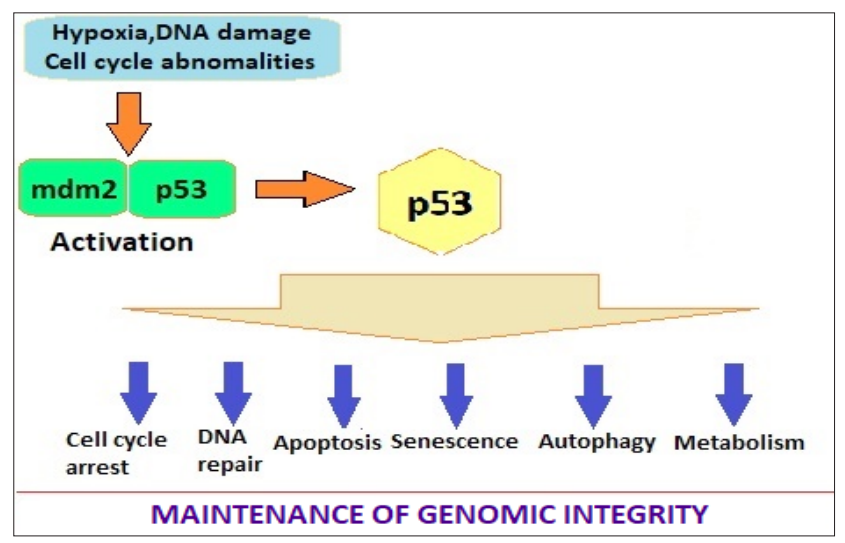

Figure 2: The stress factors activate p53 by dissociating the mdm2-p53 complex, which influences cell cycle, DNA repair, apoptosis, senescence and aging processs [3].

There is an aging, obesity and diabetes link. The physiological and histological changes in aging organs have been associated with oxidative stress, disruption of homeo- static pathways, genetic instability and telomere shortening. Diabetes, too, accelerates aging through certain complex mechanisms, which include inflammatory pathways. There are similarities in metabolic dysfunction and dysregulation in diabetes and aging. Adipose tissue from diabetic shows senescence-like changes. Both are associated with oxidative injury and impairment of insulin secretion \& sensitivity.

\section{The metabolic contributors to aging and disease}

Aging, as such, is characterized by a deterioration in the maintenance of homeostatic processes over time, leading to functional decline and increased risk for disease. Metabolically, the aging process is harbinger of IR, changes in body composition and physiological decline in growth hormone (GH), insulin-like growth factor-1 (IGF-1), and sex steroids. There occurs mitochondrial decline and endoplasmic membrane abnormalities with aging. In humans, increased adiposity typically develops between the third and seventh decades of life and may increase, decrease, or remain unchanged thereafter. With age, there is a decrease in 
subcutaneous fat (SF) and increase in VF, which is the sum of fat depots in the abdominal cavity. VF accumulation is associated with IR and development of T2DM. The SF and VF are physiologically distinct in terms of gene expression and their secretory profiles for adipokines and inflammatory markers, including leptin, TNF- $\alpha$, IL- 6 and plasminogen activation inhibitor-1, which contribute to pathogenesis of IR and associated metabolic disorders. Further, the expression of adipokines from adipose tissue is regulated by nutrients, which is exaggerated with aging. The adipose tissue derived hormone, adiponectin is another metabolic regulator, and as opposed to other fat-derived cytokines that oppose insulin action, adiponectin is an insulin sensitizer with anti-inflammatory properties and a potent activator of AMP-activated protein kinase (AMPK).

The studies have linked MetS and obesity to tendency for cognitive functional decline. These patients are more likely to have small infarcts and develop vascular dementia as occurs in hypertensive patients. The IR-associated hyperglycemia per se produces cognitive dysfunction and the cognitive decline improves when glucose is lowered. The causes of cognitive decline in MetS and T2DM are complex and multifactorial. The MetS predisposes to Alzheimer's disease. The small vascular infarcts lead to cytokine production, which may stimulate beta-amyloid production, and low testosterone levels that occurring in elderly and with T2DM may be associated with overproduction of $\beta$-amyloid, which appears to be central to the pathogenesis of Alzheimer's disease. The hyperglycemia and hypertriglyceridemia accelerates free radical production and damage to the DNA and interacting with the $\beta$-amyloid potentiate cognitive decline [5].

Skeletal muscle loss or sarcopenia is a major contributor to the frailty syndrome of aging and leads to reduced mobility and increased disability. It represents an unfavorable phenotypic change associated with aging and linked to a reduction in energy expenditure and IR. The causes of sarcopenia are multifactorial, but the proinflammatory state associated with aging and obesity is a major contributor. IR leads to the decline in muscle quantity and quality and is linked to reduced skeletal muscle strength, reduced protein synthesis rates, and accelerated skeletal muscle loss. Thus, IR is both a cause and consequence of sarcopenia, leading to a vicious cycle of skeletal muscle loss and metabolic dysfunction.

\section{The Concept of Calorie Restriction}

\section{Nutritional needs during middle age and later}

The energy needs of individuals are determined by their body composition, especially the fat free lean mass and level of physical activity. Most older adults lose fat free mass as they age, with skeletal muscle being lost at a rate of approximately $1 \%$ per year in the over 70s [6] and many are less physically active [7]. Therefore, older adults have lower requirements for energy which may contribute to a reduction in appetite. This, though, varies between individuals reflecting differences in their body composition and levels of physical activity. Further, there is a change in nutritional needs during the middle age and later, when no actual growth is taking place, though there is an increased need of nutrients to take care of increased wear and tear with age, but overall calorie requirements are less because of a sedentary nature of activity of daily living (ADL). The diet-gene interaction is a major determinant of health and illness [8]. The amount and type of food ingestion and caloric intake influence the general health and life span [9]. The extra calorie intake due to availability of palatable food and increased consumption causes overnutrition and nutritional overload [10], giving rise to excess stores in adipose tissue reservoirs culminating as weight gain and obesity, which lead to insulin resistance (IR), metabolic syndrome (MetS), type 2 diabetes mellitus (T2DM) and other metabolic alterations. At the subcellular and cellular level there is increased potentially damaging exposure to reactive oxygen species (ROS). The calorie restriction, or caloric restriction, or energy restriction, is a dietary intervention that should reduce calorie intake without incurring malnutrition or a reduction in essential nutrients [11]. The calorie restriction (CR) has been generally defined as consumption of nutritious diet that is about $40 \%$ less in calories compared to ad libitum diet. An optimal food intake and calorie restriction with adequate nutrition (CRAN) promotes health, metabolic homeostasis, disease protection and long life, in general. The focus of positive lifestyle changes is often on a healthy diet and adequate exercise to minimize the risk of diseases like diabetes, hypertension and cardiovascular disease especially during the middle age and older years. The modality, CR is like going a step further.

\section{Physiological effects of calorie restricted diet}

Several metabolic and genetic pathways have been identified that govern food ingestion, metabolism, and life span [12]. Various studies in yeast, fruit fly, nematodes like C elegans, rodent models and primates (including Homo sapiens) endose that a diet adequately fulfilling nutritional needs, but low in calories may improve health and extend the life span [13]. Experimentally, the food restriction has been shown to achieve increased lifespan in a broad spectrum of life forms from yeast [14] to primates [15]. The early nutritional studies in rodents indicated that the number of calories in the diet was the key factor, thus the term calorie restriction [16]. At the physiological level, the effects of CR are very well characterized, beginning with an acute phase upon imposition of the diet followed by an adaptive period of several weeks to reach a stable, altered physiological state [17]. A lower body temperature, lower blood glucose and insulin levels, and reduced body fat and weight characterize this altered state. The CR animals also appear to be more resistant to external stressors, including heat and oxidative stress [18].

Evolutionarily, CR may represent adaptation to food scarcity. Any organism that could slow aging and reproduction in times of scarcity and remain able to reproduce when food reappeared would enjoy an advantage [19]. In lower organisms, this strategy may lead to building of specialized body forms for survival, for example spores in microbes and dauer larvae in C Elegans [20]. The animals have reduced energy stores, i.e., glycogen and fat. About the change in metabolic rate, the initial studies reported a reduction 
in metabolic rate in CR animals. This finding fits well with the theory that oxidative damage from reactive oxygen species (ROS) is reduced, and a reduction in metabolic rate would decrease ROS production during electron transport and respiration. However, other studies found that the metabolic rate, when normalized to the lean body mass of the animals, did not decrease during CR [21]. In the studies, the CR animals enjoyed a higher metabolic activity adjusted for body weight over their lifetimes than did the ad libitum controls [22]. In budding yeast, as well as the nematode C Elegans, CR has been shown to actually result in an increase in respiration $[23,24]$. One of the most striking features of CR is that it appears to forestall or prevent many late-onset disorders and diseases. For example, CR extends life span in certain lab strains of mice which normally would die of cancer. Thus, CR extends the shortened life span of p53-/- mice which otherwise die of early cancers that appear in the absence of the tumor suppressor [25,26]. CR also extends life span in Fischer rats, which normally die of kidney disease. Further, CR has been shown efficacious in mouse models of a variety of diseases $[27,28]$.

\section{The Cr mechanisms and pathways}

\section{The sirtuins and allied pathways}

It is important to understand that the benefits of CR are not a passive result of lower caloric intake but the consequence of an active regulatory intervention mimicking the food scarcity activating certain genetic and metabolic programs that result in beneficial vital effects. Various genetic and molecular studies in model organisms, in fact, suggest that CR is a regulated process, in which the SIRT (Silent Information Regulator 2) gene plays an important role. The SIR2 gene was first identified and so-named because it mediates gene silencing in yeast [29]. The findings suggest that the SIR2 ortholog, Sirt1 in mammals may mediate a broad array of physiological effects that occur in animals on a CR diet. Sirtuin 1, also known as NAD-dependent deacetylase sirtuin-1, is a protein encoded by the SIRT1 gene.

The elevated activity of SIRT1 orthologs has been shown to extend life span in yeast (Saccharomyces cerevisiae), nematodes (Caenorhabditis elegans) and fruit flies (Drosophila melanogaster). The CR also increases SIRT1 levels in mice, rats, non-human primates and humans. The regulation of SIRT1 activity by CR is complex, being tissue-specific as well as region-specific in nonhomogeneous tissues, such as the brain [30]. The activity of the sirtuin in the liver is reduced by CR and correlates with the reduced fat synthesis and is activated by a high-caloric diet. The SIRT1, thus figures prominently in the redistribution of resources during CR from growth, metabolism and reproduction to maintenance and survival.

The related genes in S Cerevisiae, C Elegans and D Melanogaster called sirtuins, encode NAD-dependent deacetylases and appear to promote longevity in the organisms [31]. In the mammals there are at least seven sirtuins (SIRT1-7), each sirtuin influencing diverse aspects of the metabolism and characterized by differences in subcellular localization, substrate preference, and biological function. In the study models ranging from yeast to mice, sirtuins have also been associated with the salutary effects of CR. The mammalian Sir2 ortholog SIRT1 targets numerous regulatory factors affecting stress effects to metabolism [32]. The levels of SIRT1 increase in rodent and human tissues in response to CR leading to favorable changes in metabolism and stress tolerance [33]. The SIRT1 promotes oxidation of fatty acids in liver and skeletal muscle, cholesterol metabolism in liver, and lipid mobilization in white adipose tissue [34]. Sirt1 may also regulate CR by sensing low calories and triggering physiological changes linked to health and longevity [35]. The gene, SIR2, encodes an NAD-dependent deacetylase and may mediate the effects of CR, as shown by experiments in the lower organisms, yeast and Drosophila. Further, the small-molecule activators of SIRT1 have been shown to protect mice from the negative effects of a high-fat diet.

\section{The CR mechanisms}

The CR appears to work through the following mechanisms:

CR lowers the core body temperature: An adaptive response to reduce energy expenditure when nutrients availability is curtailed. Lowering the temperature may prolong the lifespan of cold-blooded animals. Mice, which are warm blooded, have been genetically modified to have a reduced core body temperature which increases the lifespan independently of calorie restriction.

Hormesis: The CR is a low-intensity biological stressor. The CR diet imposes a low-intensity biological stress on the organism to elicit a defensive response that help to protect from disorders of aging. The CR places the organism in a defensive state to survive in adverse life situations, resulting in improved health and longer life through activation of longevity genes. In C. Elegans, the CR extends life span primarily by increasing oxidative stress to stimulate the organism into having an increased resistance to further oxidative stress.

Hormonal alterations: Prolonged severe CR lowers total serum and free testosterone while increasing sex hormone binding globulin concentrations in humans. Calorie restriction has been shown to increases DHEA in primates, but not in post-pubescent primates. These effects are independent of adiposity. Lowering of the concentration of insulin and insulin-like growth factor 1 and growth hormone, has been shown to up-regulate autophagy, the repair mechanism of the cell. The CR works by decreasing insulin levels and up-regulating autophagy.

CR reduces production of ROS and damage by ROS. The free radicals may induce an endogenous response culminating in effective adaptations to protect against exogenous radicals. The sublethal mitochondrial stress with ROS may initiate beneficial alterations in cellular physiology produced by CR. The CR is related to chromatin function. In C. Elegans, the gene PHA-4 is responsible for the increased life span. Whereas, in rodents, CR slows aging, decreases ROS production and reduces the accumulation of oxidative DNA damage in multiple organs. These results are linked to reduced oxidative DNA damage. The CR also decreases 8-OHdG damages in the DNA of mice heart, skeletal muscle, brain, liver and kidney12. The levels of 8-OHdG in the DNA of these organs were reduced to than that in the DNA of mice fed an unrestricted diet. Also, in rats, CR retarded the onset of age-related increases in 
8-OHdG in nuclear DNA of brain, heart, liver and kidney. The level of $8-0 H d G$ in these organs of the calorie restricted rats at 30 months averaged $65 \%$ of the level in rats fed an unrestricted diet.

Sirtuins: Sir2 has been implicated in the aging of S. cerevisiae and is a highly conserved, NAD+-dependent histone deacetylase. Sir2 homologs have been identified in a wide range of organisms from bacteria to humans. Yeast has 3 SIR genes - SIR2, SIR3, and SIR4. Although all three genes are required for the silencing of mating type loci and telomeres, only SIR2 has been implicated in the silencing of rDNA. In addition, SIR2 related genes also regulate formation of some specialized survival forms, such as spores in S. cerevisiae and dasher larvae in C. Elegans. A study done by Kaeberlein et al. [36] in yeast found that deletions of Sir2 decreased lifespan and its additional copies increased lifespan.

CR mimetics: the results akin to $\mathrm{CR}$, can be achieved with pharmacologic approaches, such as rapamycin, via mTOR signaling blockade, resveratrol, by activating SIRT1 activity, and metformin, which seems to be a stimulator of AMPK activity. The polyphenol resveratrol partially mimics CR by activating SIRT1 to induce beneficial effects on health $[37,38]$.

\section{The CR studies in animal models}

CR in $\mathbf{S}$ cerevisiae and $\mathbf{C}$ elegans: The classic experiments, diluting the energy source in growth media, namely glucose, extended the replicative life span of yeast mother cells [39]. This intervention extended the life span, by mutating genes for glucose utilization (hexokinase) and/or signaling components of the protein kinase A pathway. The major genetic determinant of replicative life span in yeast is SIR2 and its increased activity extended it [40]. The SIR2 ortholog in C Elegans was also found to be the key determinant of life span. The evolutionary studies highlight that S. cerevisiae and C Elegans diverged from a common ancestor about a billion years ago and ratify that all descendants of that common ancestor, including mammals, have conserved the SIR2-related genes involved in regulating life span.

The phylogenetically conserved enzymatic activity of the SIR2 and its homologs is determined by NAD-dependent protein deacetylases [41]. The studies show that the mammalian Sirt1 enzyme deacetylates many histone and nonhistone substrates, and NAD is cleaved to produce nicotinamide (NA) and acetylADP-ribose each reaction cycle [42]. The studies suggest that Sir2 senses the metabolic state of cells and sets the life span accordingly [43]. Further, the SIR2-related genes regulate formation of the specialized survival forms in lower organisms, spores in yeast [44] and dauer larvae in C elegans [45]. Several studies suggest that CR in yeast is regulated by SIR2 [46] and CR increased the silencing activity of SIR2 in vivo [47]. Also, CR did not extend the life span when SIR2 was deleted. Further, an activator of the Sir2 enzyme, resveratrol, has been shown to extends yeast replicative life span [48]. Resveratrol requires the SIR2 gene for this longevity effect. Activating SIRT1 pathway might be beneficial in preventing some manifestations of aging (Figure 3). But resveratrol and CR did not synergize to further extend the life span, suggesting that CR and resveratrol act through the same pathway.

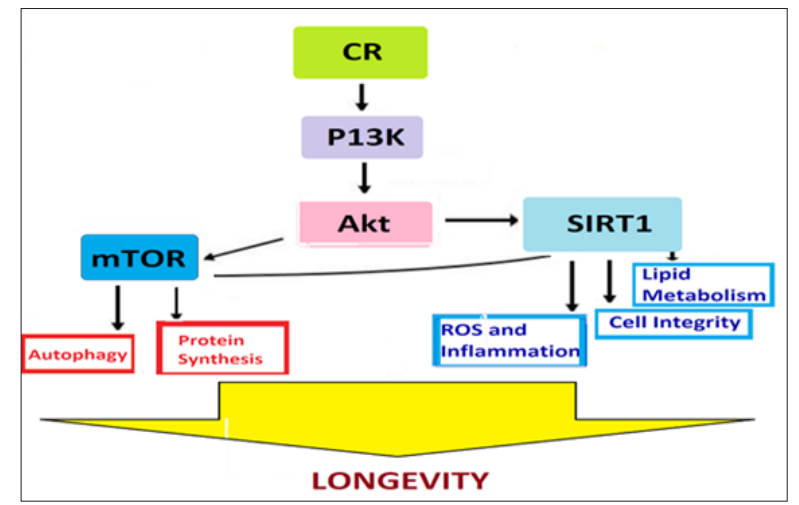

Figure 3: CR, SIRT1 and Life Span: Projected Metabolic Pathways.

Resveratrol is a plant-derived polyphenol that appears to activate SIRT1 apart from having antioxidant, anti-inflammatory, and antitumorigenic properties. Through activation of SIRT1, resveratrol may function as a CR mimetic and resveratrol treatment has been shown to increase life span in several organisms, including, high-fat-fed mice, in which it improved insulin sensitivity, mitochondrial function, and survival. More recently, treatment of obese mice with SRT1720, a synthetic activator of SIRT1, resulted in similar improvements in survival as were observed in resveratroltreated mice. The mechanism by which CR activates the Sir2 enzyme is not fully understood, though a molecular pathway could be traced from caloric intake. In S. cerevisiae, the fermentation is a way for cells to generate ATP and to store excess energy in the form of ethanol when glucose is abundant. NADH itself is a competitive inhibitor of SIR2 and its reduction during CR upregulates the enzyme to extend the life span.

The CR triggers a more efficient use of glucose via an increase in respiration, analogous to a known metabolic shift that occurs in mammals during CR, in whom there is a transition in muscle cells from using glucose towards the use of fatty acids. This metabolic shift spares glucose for the brain and correlates with the characteristic enhancement of insulin sensitivity in muscle and liver. But, this model for activation of Sir2p by CR in yeast, however, is not established. Instead, the NAD salvage pathway may play a key role in CR. The CR upregulates levels of an enzyme, Pnc1p, that synthesizes NAD from its cleaved products, NA and ADP-ribose. Since NA has also been shown to inhibit the SIR2 enzyme, it has been suggested that CR extends life span by reducing NA in cells, thereby upregulating Sir2p. Consistent with this model, deleting PNC1 reduced or eliminated the ability of CR to extend life span [49]. As the synthesis of the two theories, it is possible that the NAD salvage pathway functions in parallel to changes in NADH to regulate Sir2. To validate this, NA levels are in fact altered in CR cells. However, that NA functions as the mediator of CR, since the regimen was completely effective in cells deleted for PNC1 if the excess NA was depleted from cells [50]. In another study, it was reported that CR is independent of SIR2 in a different, unrelated strain of yeast [51]. This finding endorses that there are than one pathway that mediate CR in yeast. 
CR in Drosophila: The life span in Drosophila can be extended by diluting the fruit fly's diet comprising of yeast along with glucose [52]. This form of food restriction works by activating the SIR2 ortholog, Sir2, and increasing levels of Sir2 mRNA in Drosophila. These observations suggest the role of SIR2 as mediator of CR for life span, which has been conserved in metazoans. The SIR2 activator, resveratrol also extends the life span in Drosophila [53] but does not further extend the longer life span of CR flies, indicating that Sir2 works in the same pathway as CR [54]. In study models, certain mutations in the insulin/IGF-1 signaling pathway appear to extend the life span in C Elegans, flies and mice [55]. The CR does not extend the life span of Sir2 mutant flies. But, the longlived dwarf mice, with missing the growth hormone-IGF-1 axis and other pituitary hormones due to mutation in the pit- 1 gene, shows further extension in their life span on a low-calorie diet [56]. These finding leads to inference that CR and IGF-1 may extend life span by independent mechanisms. Thus, the effect of CR in mammals is more complex than C. Elegans and Drosophila, involving various organs and physiological axes. Thus, the metabolic pathways do not stand alone, but are interconnected and merge at various levels. In mammals, the CR appears to affect metabolism and studies suggest that it may be advantageous to increase metabolism during CR, at least in some tissues to promote a longer lifespan in mammals.

\section{Metabolic alterations with $\mathrm{Cr}$}

Upregulation of mitochondrial uncoupling proteins: The mice are suitable models for mammalian studies concerning effect of CR and metabolic and genetic alterations on lifespan. The fat insulin receptor knock-out mice (FIRKO) lacking insulin signaling, have low fat deposits in white adipose tissue (WAT). These mice eat more than weight-matched wild-type mice yet have a lower body weight, indicating that they have a higher than normal metabolic rate. Further, these mice live longer than controls [57]. Another group of genetically engineered mice having their $\mathrm{C} / \mathrm{EBP} \alpha$ gene replaced with a second copy of $\mathrm{C} / \mathrm{EBP} \beta$ are also hypermetabolic due to increased metabolism in WAT. These mice also live longer than controls [58].

Another study in mice showed a positive correlation between oxygen consumption, i.e., metabolic rate, and life span [59]. In this study, the variation in metabolism was due to differences in the degree to which electron transport was coupled to ATP synthesis in mitochondria. The longer-lived mice had mitochondria that were more uncoupled. Also, the CR mice have more uncoupled mitochondria than controls, perhaps due to upregulation of mitochondrial uncoupling proteins by $\mathrm{CR}$. CR reduces the size of animals, likely creating a higher surface area and potentially greater heat loss, and thus the lower body temperature. The CR mice may increase uncoupling proteins in metabolic tissues, muscle, liver, and brown adipose tissue. It appears that the extra electron transport dissipated by uncoupling may activate a regulatory pathway to extend life span. Because of proton leakage down the gradient, uncoupled mice would avoid hyperpolarization of the mitochondrial membrane and partially uncoupled electron transport may generate a lower level of ROS.
CRand stress resistance: $C R$ is known to increase the resistance to oxidative stress [60], which leads to longer life span by a greater ability to detoxify ROS and repair oxidative damage, and slow down cellular decay [61]. The genetic regulators of mammalian life span can also increase resistance to stress. Absence of the protein p66 shc causes an increase in stress resistance and the KO mice and cells from $\mathrm{KO}$ mice are more resistant to oxidative stress, and the p66 K0 mice live longer than wild-type. Experimentally, in vitro, the CR serum triggers a higher level of the mammalian SIR2 ortholog, Sirt1, in the fibroblasts, which is partially reversed by adding IGF-1 and insulin to the serum.

Further, the connection between Sirt1 and stress resistance appears to be extensive. The Sirt1 is an NAD-dependent deacetylase and appears to target many proteins that are not histones and an important one, p53, which is shown to be deacetylated and downregulated by Sirt1 [62]. Thus, Sirt1 negatively regulates p53dependent apoptosis in response to cellular damage [63]. Another family of regulators targeted by Sirt 1 are Foxo or forkhead proteins, which, like p53, can respond to stress and trigger apoptosis. The Sirt1 was shown to deacetylate and downregulate Foxo1, 3, and 4 and thus repress Foxo-mediated apoptosis [64]. Sirt1 also deacetylates and downregulates the Foxo coactivator p300. In addition, Sirt1 deacetylates the DNA repair protein Ku70, allowing it to bind to and inactivate the proapoptotic factor Bax [65]. Thus, Sirt1 appears to target numerous cellular factors, thereby resulting in a higher threshold for apoptosis [66].

CR and fat regulation: There are increasing evidence that mammalian aging is regulated in part by adiposity [67]. The WAT stores fat as triglycerides when food is abundant. When food is scarce, as in CR animals, cells shed their fat from adipose tissue. The WAT can sense nutritional status and sends appropriate signals to coordinate aging in all organs, and in addition, WAT is an endocrine organ and secretes hormones such as leptin and adiponectin [68]. The idea that WAT regulates aging is strengthened by the findings that the FIRKO mice and C/EBP knockin mice stay lean and live long. The WAT also mediates many age-associated metabolic disorders such as T2DM and dyslipidemia which can negatively affect the lifespan [69]. The Sirt1, thus, regulates WAT by repressing p53. It exerts effects on hormones, including growth factors, which override cell-autonomous effects on apoptosis. Such changes may permit or even mandate raising the threshold for apoptosis in hormone-responsive cells. The combined effect of changes in hormones and a higher threshold for apoptosis in responsive cells is an advantage for the survival.

The WAT, muscle, liver and pancreatic $\beta$ cells are integrated in a regulatory circuit and an important hormonal axis during CR comprises WAT, metabolic organs (muscle and liver) and pancreatic $\beta$ cells producing insulin. Chronic high food intake triggers an increase in blood glucose. The surges in blood glucose will activate $\beta$ cells to produce more insulin, in the long run leading to $\beta$ cell proliferation and ultimately failure. Insulin signals WAT to store fat as triglycerides. The triglycerides store influences the levels of hormones produced by the WAT cells, primarily increase in leptin 
and a decrease in adiponectin [70]. These hormonal changes influence the insulin sensitivity of metabolic organs. Adiponectin increases sensitivity to insulin in metabolic organs and its decrease exacerbates insulin resistance and rise in blood glucose [71]. This vicious cycle initiated by adiposity and culminating as T2DM (Figure 4), is antagonized by CR by lowering blood glucose and insulin, reducing fat stores, increasing adiponectin and improving insulin sensitivity.

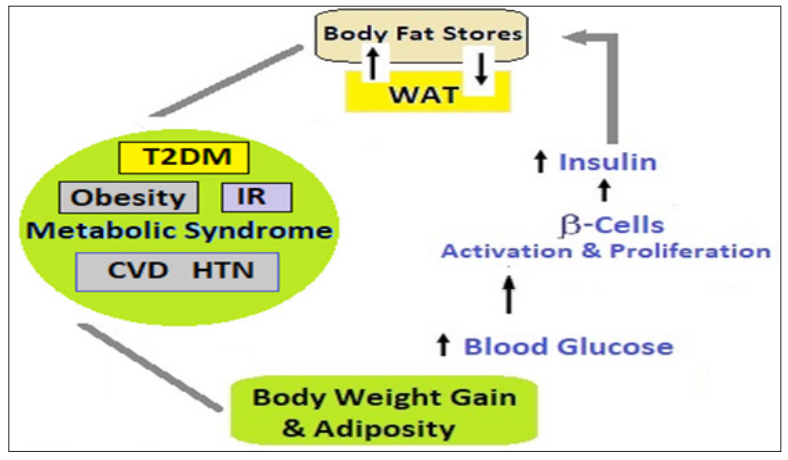

Figure 4: Metabolic Changes Triggered by Adiposity - The links between Aging, visceral fat, inflammation and MetS. Visceral fat increases with age and its increase induces inflammation. Inflammation accelerates process of aging. The aging, visceral fat and inflammation increase risk of metabolic diseases like obesity, IR, T2DM, CVD and HTN.

In WAT, Sirt1 is expressed and represses the key regulator of WAT, the nuclear receptor PPAR $\gamma$, which slows down the differentiation of precursor cells into white adipocytes and downregulates fat storage in existing WAT [72]. Sirt1 is bound directly to the PPAR $\gamma$-negative cofactors, NCoR and SMRT, and associated with PPAR $\gamma$ DNA binding sites in promoters of fat-specific genes, and functions as a negative regulator of PPAR $\gamma$. The CR causes the lipolysis of triglycerides in WAT and the release of free fatty acids, which are taken up and oxidized by metabolic organs. Further, CR induces the binding of Sirt1 to the PPAR $\gamma$ DNA binding region of the promoters of fatspecific genes to exert repressive effects. The CR, thus, increases levels of Sirt1 [73], which modulates the genes concerned with fat storage and hormones. The changes in Sirt1 during long-term CR, by increasing $\beta$ oxidation of fatty acids and lowering free fatty acids, improve insulin sensitivity, and working in a coordinated way lead to slowing of aging. The Sirt1-PPAR $\gamma$ connection is important in other tissues as well. It controls many biological processes in the vessel wall, such as smooth muscle cell proliferation or differentiation and lipid accumulation in macrophages [74], playing an active role in age-related atherosclerosis.

CR and neurodegeneration: In certain mouse models of neurodegenerative diseases, such as Parkinson's or Alzheimer's, CR has been reported to reduce age-associated neuronal loss [75]. CR has also been reported to slow declines in psychomotor and special memory tasks, preserve dendritic spines involved in learning, and improve the plasticity and ability of self-repair of the brain [76]. The repression of p53- and FOXO-mediated apoptosis by Sirt1 may be of special importance for the long-term maintenance of postmitotic cells such as neurons. There appears to be a connection between Sirt and the preservation of neuronal integrity [77]. In the neuronal cell body, Sirt1 regulates genes whose products travel down the axon and mediate axonal survival in event of injury.

\section{Impact of $\mathrm{Cr}$ on health and disease}

CR has long been recognized for its ability to extend life span and to mitigate aging and disease processes in various tissues. The effects of CR with adequate nutrition seem to possess many beneficial effects in retarding numerous disease states [78]. The studies related to calorie restriction mimetics (CRMs), number of phytochemicals that biochemically mimic the effects of CR, prove that both CR and CRMs trigger an adaptive response similar to mild-stress or a low-dose response, referred to as hormesis [79]. Both CR and CRMs affect a common pool of biochemical pathways that are involved in an organism's survival and longevity [80]. The two bio-metabolic pathways, sirtuin pathway and Kelch-like ECHassociated protein 1 (Keap1)/nuclear factor erythroid 2-related factor 2 (Nrf2)/antioxidant response element (ARE) pathway, also referred to as the Keap1/Nrf2/ARE pathway are concerned with an organism's longevity and upregulation of cytoprotective genes essential for cell survival respectively [81].

\section{Studies of $\mathrm{Cr}$ effects in animal models}

During 1935, based on his classical animal studies at Cornell University, Clive McKay proved that a $40 \%$ calorie reduced diet prolonged the life in mice [82]. The findings have since been experimented in other animals and there exists a possibility of parallel physiological links in non-human primates and humans [83]. In a 2017 collaborative report on rhesus monkeys by scientists of the US National Institute on Aging and the University of Wisconsin, CR in the presence of adequate nutrition was effective in delaying the effects of aging [84]. The older age, female sex, lower body weight and fat mass, reduced food intake, diet quality, and lower fasting blood glucose levels were factors associated with fewer disorders of aging and improved survival. The reduced food intake was beneficial in adult and older primates, but not in younger monkeys. Because rhesus monkeys are genetically similar to humans, the benefits and mechanisms of CR may apply to human health and aging. Earlier, the NIA rhesus macaque calorie restriction study in 1987 had shown that CR did not extend years of life or reduce age-related deaths in rhesus monkeys. It did improve certain measures of health but did not have any significant effect on life-span. The CR represents an important intervention to extend both mean and maximum life span in various organisms. This is due to numerous metabolic alterations following CR (Figure 5). The CR preserves muscle tissue in nonhuman primates and rodents. Mechanisms include reduced muscle cell apoptosis and inflammation, protection against age-related mitochondrial abnormalities and preserved muscle stem cell function [85]. Laboratory rodents placed on a CR diet tend to exhibit increased activity levels at feeding time. Monkeys undergoing CR also appear 
more restless immediately before and after meals, and have demonstrated an increase in licking, sucking, and rocking behavior.
The CR regimen may also lead to increased aggressive behavior in animals [86].

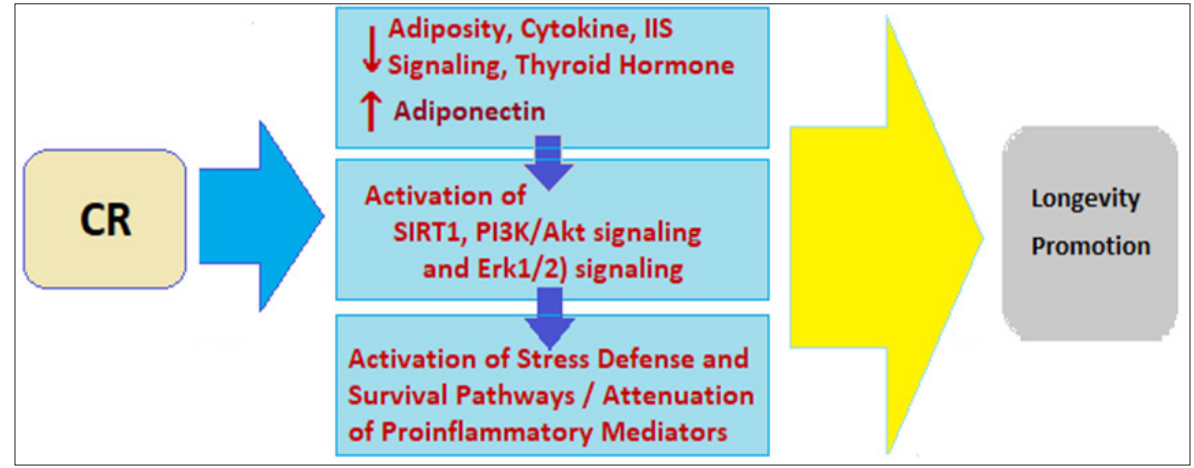

Figure 5: CR and Cellular Pathways for Longevity Promotion - Various metabolic alterations in Body fat mass, Cytokines, IIS signaling, Thyroid hormones and Adiponection levels, accompanied by Activation of SIRT1, PI3K/Akt and Erk 1/2 signaling, and Activation of Stress defense plus Survival Pathways and Attenuation of Proinflammatory mediators.

\section{The CR and age-related disease risk}

Data from animal and human studies indicate that the effects of CR and especially CRAN on the aging heart and vasculature may have additional beneficial effects on several metabolic and molecular factors that modulate cardiovascular aging alterations including cardiac and arterial stiffness and heart rate variability [87]. The risk factors for atherosclerosis are substantially improved in a manner consistent with experimental studies in rodent models of atherosclerosis and nonhuman primates. The risk factors such as c-reactive protein, serum triglycerides, low-density lipoprotein, high-density lipoprotein, blood pressure and fasting blood sugar, are substantially more favorable with CR than with unrestricted dietary schedule and comparable with the long-term endurance exercise plan [88].

The risk factors for atherosclerosis are substantially improved in a manner consistent with experimental studies in rodent and nonhuman primate models of atherosclerosis. Other risk factors for CVD also become substantially more favorable. Similar effects were also seen during a natural experiment in Biosphere 2, and effects on blood pressure, cholesterol level, and resting heart rate were seen in subjects in the Minnesota Starvation Experiment, during World War II [89]. The cardiac function parameters including the diastolic function were found to be better in subjects who practiced CR for 3-15 years than that in healthy age- and sex-matched control subjects. The CR subjects had less ventricular stiffness and less viscous loss of diastolic recoil, both of which would be consistent with less myocardial fibrosis.

These effects, in combination with other benefits of calorie restriction, such as protection against obesity, diabetes, hypertension, and cancer, suggest that CR may have a major beneficial effect on health span, quality of life and life span in humans. Further, a detailed review of the effects of CR on the aging heart and vasculature concluded that the data from animal and human studies indicate that beyond the effects of implementation of healthier diets and regular exercise, CRAN may have additional beneficial effects on several metabolic and molecular factors that are modulating cardiovascular aging including cardiac and arterial stiffness and heart rate variability.

\section{Conclusion: CR in Practice}

\section{Metabolic strategy to delay aging process}

Biological characteristics of animals following CR include numerous changes in the transcriptome, metabolome, and proteome, as well as increase in stress hormones. CR leads to decline in insulin, thyroid hormone, reproductive hormones and GH/IGF-1 levels. Some of these effects represent restoration to levels similar to that in younger age, such as lower insulin and glucose concentrations, whereas other changes resemble those in older age, such as low GH/IGF-1. Because CR modulates a remarkable number of biologic systems and it appears that the the longevity-promoting effects of CR are elicited by concurrent interplay of several mechanisms. The Akt activation of NF- $\kappa \mathrm{B}$ may be mTOR-dependent, whereas SIRT1 may be a direct stimulator of AMPK activity and autophagy.

\section{The CR studies across the species}

Most CR studies have been conducted in rats and mice, but CR experiments in other mammalian species as well as taxonomically distant organisms suggest the universality of its beneficial effect on life span. In nonhuman primates, CR decreases the risk of T2DM and other age-related diseases and appears to extend life span. Most of the effects of CR in animal studies have been reproduced in middle-aged humans and it is capable of reducing diseasespecific mortality risk, though the issue about life span extension in humans remains un-resolved. The possible benefits of CR should be carefully weighed against the QOL concerns. The middle-aged individuals subjected to rigorous CR may experience issues with low bone density and muscle mass, and feel hungry, muscular pains, lethargic and cold [90,91]. Thus, CR as a late-life intervention will be counterproductive in frail, sarcopenic individuals in whom adequate caloric and protein intake are essential to maintaining 
bone and muscle mass. Some of these concerns can be overcome by using a more moderate CR regimen with adequate essential vitamins and minerals supplementation, and a suitable exercise program. Such a strategy has been shown to promote improve several health indices while simultaneously preserving bone mineral density, lean body mass, strength, and aerobic capacity.

\section{The CR and role of genetics}

The somatotropic signaling: The GH/IGF-1 axis declines with aging. Life span extension has also been demonstrated in mutant mice with reduced function of the somatotropic axis, including Ames and Snell dwarf mice and mice lacking the GH receptor, all of which have decreased plasma IGF-1 concentrations. The extended longevity is due to GH deficiency, as restoration of GH levels in Ames dwarf mice reverted their longevity to that of nonmutant controls. In addition, functional mutations have been identified in the human IGF-1R gene that result in altered IGF-1 signaling and are common in centenarians. Both animal and human studies have also linked reduced IGF-1 levels/signaling per se with reduced risk of many cancers as well as improved longevity, but the low IGF-1 concentration in humans carries an increased risk for CVD, stroke, T2DM, and osteoporosis.

Sirtuins, AMPK activators and mTOR signaling: The sirtuin family of proteins is a seven-member group (SIRT1-7) of highly conserved, nicotinamide adenine dinucleotide-dependent protein deacetylases that function in the regulation of various metabolic and biological processes. The mammalian SIRT1 shares the closest similarity to Sir2, plays an important role in regulating glucose metabolism, insulin action, fat storage, and nutrient sensing. It deacetylates the inflammatory regulator nuclear factor- $\mathrm{kB}$ (NF$\kappa B)$, which is a key player in IR and the MetS. Activating SIRT1 pathway through CRMs may be beneficial in preventing several manifestations of aging including improved insulin sensitivity, mitochondrial function, and cell integrity and survival.

AMPK plays a critical role in regulating whole-body energy balance and is activated by various interventions such as exercise or CR. In the hypothalamus, AMPK activation in agouti-related peptide or pro-opiomelanocortin neurons stimulates food intake, whereas activation of AMPK in muscle promotes glucose transport, fatty acid oxidation, and mitochondrial biogenesis. The AMPK activators can serve as exercise or CR mimetics. In sedentary mice, the 4 weeks of treatment with the AMPK agonist, AICAR, substantially enhanced running endurance. Similarly, metformin, which is an AMPK activator, increases life span in yeast and mice, though not in rats. Further, there are no data to support a role for metformin in human aging, which is well-tolerated and have anti-diabetic and anticancer property, as well. The mechanism by which AMPK activation modulates aging and disease risk is not established.

The mTOR signaling pathway is highly conserved and integrates energy and growth factor signaling to cell growth and basic cellular processes such as RNA translation, stress resistance, and autophagy. It is closely linked to components of IIS pathways, energy metabolism and glucose homeostasis. The inhibition of mTOR signaling pathway by genetic or pharmacological intervention extends life span in yeast, nematodes, and fruit flies. Also, the inhibition of the downstream effector of mTOR, S6K, increases life span in worms and flies and protects against dietinduced obesity and enhances insulin sensitivity in mice. The pharmacologic inhibition of mTOR with rapamycin has also been shown to extend life span in mice. In mice, the specific deletion of raptor, a component of mTOR complex in adipose tissue, protects against diet-induced obesity, whereas deletion of raptor in skeletal muscle results in muscular dystrophy. The inhibition of mTOR in the pancreas decreases insulin production by islets, and increased mTOR activity in the brain or the hypothalamus leads to decreased appetite via modulation of leptin and ciliary neurotrophic factor. The human skeletal muscle from older adults has been shown to have impaired mTOR complex 1 activation and systemic blockade of the mTOR complex 1 pathway eliciting an aging skeletal muscle phenotype, sarcopenia and frailty.

\section{References}

1. Morley JE (2004) The metabolic syndrome and aging. J Gerontol A Biol Sci Med Sci 59(2): 139-142.

2. Dirks AJ, Hofer T, Marzetti E (2006) Mitochondrial DNA mutations, energy metabolism and apoptosis in aging muscle. Ageing Res Rev 5(2): 179-195.

3. Nikhra V (2014) 'ERDOMA: The entangled relationship between diabetes, obesity, metabolic syndrome and aging'. Cardiology Today 18(4): 157-166.

4. Minamino T, Orimo M, Shimizu I (2009) A crucial role for adipose tissue p53 in the regulation of insulin resistance. Nat Med 15(9): 1082-1087.

5. Barzilai N, Huffman DM, Radhika H (2012) The critical role of metabolic pathways in aging. Diabetes 61(6): 1315-1322.

6. Goodpaster B, Park S, Harris T (2006) The loss of skeletal muscle strength, mass, and quality in older adults: the health, aging and body composition study. J Gerontol A Biol Sci Med Sci 61(10): 1059-1064.

7. Taylor A, Cable N, Faulkner G (2004) Physical activity and older adults: a review of health benefits and the effectiveness of interventions. Journal of Sports Sciences 22(8): 703-725.

8. McKay JA, Mathers JC (2011) Diet induced epigenetic changes and their implications for health. Acta Physiol (Oxf) 202(2): 103-118.

9. Koubova J, Guarente L (2003) How does calorie restriction work? Genes Dev 17(3): 313-321.

10. James PT, Rigby N, Leach R (2004) The obesity epidemic, metabolic syndrome and future prevention strategies. Eur J Cardiovasc Prev Rehabil 11(1): 3-8.

11. Wolf G (2006) Calorie restriction increases life span: a molecular mechanism. Nutrition reviews 64(2): 89-92.

12. Kenyon C (2005) The plasticity of aging: Insights from long-lived mutants. Cell 120(4): 449-460.

13. Sinclair DA (2005) Toward a unified theory of calori c restriction and longevity regulation. Mech Ageing Dev 126(9): 987-1002.

14. Weindruch R, Walford RL (1988) The retardation of aging and disease by dietary restriction. In: Charles C Thomas (Ed.), Springfiled, USA.

15. Lin SJ, Defossez PA, Guarente L (2000) Requirement of NAD and SIR2 for life-span extension by calorie restriction in Saccharomyces cerevisiae. Science 289(5487): 2126-2128.

16. Ingram DK, Anson RM, de Cabo R (2004) Development of calorie restriction mimetics as a prolongevity strategy. N Y Acad Sci 1019: 412423. 
17. Masaro EJ (1984) Nutrition as a modulator of the aging process Physiologist 27: 98-101.

18. Sohal RS, Weindruch R (1996) Oxidative stress, caloric restriction and aging. Science 273(5271): 59-63.

19. Harrison D (1989) Natural selection for extended longevity from food restriction. Growth Dev Aging 53(1-2): 3-6.

20. Holliday R (1989) Food, reproduction, and longevity: is the extended life span of calorie-restricted animals an evolutionary adaptation? Bioessays 10(4): 125-127.

21. McCarter R, Masoro EJ, Yu BP (1985) Does food restriction retard aging by reducing the metabolic rate? Am J Physiol 248(4): E488-E490.

22. McCarter RJ, Palmer J (1992) Energy metabolism and aging: a lifelong study of Fischer 344 rats. Am J Physiol 263(3): E448-E452.

23. Lin SJ, Kaeberlein M, Andalis AA (2002) Calorie restriction extends Saccharomyces cerevisiae lifespan by increasing respiration. Nature 418(6895): 344-348

24. Houthoofd K, Braeckman BP, Lenaerts I (2002) No reduction of metabolic rate in food restricted Caenorhabditis elegans. Exp. Gerontol 37(12): 1359-1369.

25. Hulbert AJ, Clancy DJ, Mair W (2004) Metabolic rate is not reduced by dietary-restriction or by lowered insulin/IGF-1 signalling and is not correlated with individual lifespan in Drosophila melanogaster. Exp Gerontol 39(8): 1137-1143.

26. Hursting SD, Lavigne JA, Berrigan D (2003) Calorie restriction, aging, and cancer prevention: mechanisms of action and applicability to humans. Annu Rev Med 54: 131-152.

27. Berrigan D, Perkins S, Haines DC, Hursting S (2002) Carcinogenesis adult-onset calorie restriction and fasting delay the spontaneous tumorigenesis in p53-deficient mice. Carcinogenesis 23(5): 817-822.

28. Lane MA, Ingram DK, Roth GS (1999) Calorie restriction in nonhuman primates: effects on diabetes and cardiovascular disease risk. Toxicol Sci 52(2): 41-48

29. Stern JS, Gades MD, Wheeldon CM, Borchers AT (2001) Calorie restriction in obesity: prevention of kidney disease in rodents. J Nutr 131(3): 913S-917S.

30. Rine J, Herskowitz I (1987) Four genes responsible for a position effect on expression from HML ANS HMR in Saccharomyces cerevisiae. Genetics 116(1): 9-22.

31. Chen D, Bruno J, Easlon E (2008) Tissue-specific regulation of SIRT1 by calorie restriction. Genes Dev 22(13): 1753-1757.

32. Chen D, Guarente L (2007) SIR2: A potential target for calorie restriction mimetics. Trends Mol Med 13(2): 64-71.

33. Sinclair DA (2005) Toward a unified theory of caloric restriction and longevity regulation. Mech Ageing Dev 126(9): 987-1002.

34. Civitarese AE, Carling S, Heilbronn LK (2007) Calorie restriction increases muscle mitochondrial biogenesis in healthy humans. PLoS Med 4(3): e76.

35. Lomb DJ, Laurent G, Haigis MC (2010) Sirtuins regulate key aspects of lipid metabolism. Biochimica et biophysica acta 1804(8): 1652-1657.

36. Kaeberlein M, McVey M, Guarente L (1999) The SIR2/3/4 complex and SIR2 alone promote longevity in Saccharomyces cerevisiae by two different mechanisms. Genes Dev 13(19): 2570-2580.

37. Guarente L, Picard F (2005) Calorie restriction-the SIR2 connection. Cell 120(4): 473-82.

38. Baur JA, Pearson KJ, Price NL (2006) Resveratrol improves health and survival of mice on a high-calorie diet. Nature 444(7117): 337-342.

39. Lagouge M, Argmann C, Gerhart-Hines Z (2006) Resveratrol improves mitochondrial function and protects against metabolic disease by activating SIRT1 and PGC-1 $\alpha$. Cell 127: 1109-1122.
40. Lin SJ, Defossez PA, Guarente L (2000) Requirement of NAD and SIR2 for life-span extension by calorie restriction in Saccharomyces cerevisiae. Science 289(5487): 2126-2128.

41. Tissenbaum HA, Guarente L (2001) Increased dosage of a sir-2 gene extends lifespan in Caenorhabditis elegans. Nature 410(6825): 227-230.

42. Tanny JC, Moazed D (2001) Coupling of histone deacetylation to NAD breakdown by the yeast silencing protein Sir2: evidence for acetyl transfer from substrate to an NAD breakdown product. Proc Natl Acad Sci 98(2): 415-420

43. Tanner KG, Landry J, Sternglanz R, Denu JM (2000) Silent information regulator 2 family of NAD-dependent histone/protein deacetylases generate a unique product, 1-0-acetyl-ADP-ribose. Proc Natl Acad Sci 97(26): 14178-14182.

44. Guarente L (2000) Sir2 links chromatin silencing, metabolism and aging. Genes Dev 14(9): 1021-1026.

45. Margolskee JP (1988) The sporulation capable (SCA) mutation of Saccharomyces cerevisiae is an allele of the SIR2 gene. Mol Gen Genet 211(3): 430-434.

46. Tissenbaum HA, Guarente L (2001) Increased dosage of a sir-2 gene extends lifespan in Caenorhabditis Elegans. Nature 410(6825): 227-230.

47. Lin SJ, Defossez PA, Guarente L (2000) Requirement of NAD and SIR2 for life-span extension by calorie restriction in Saccharomyces cerevisiae. Science 289(5487): 2126-228.

48. Lin SJ, Kaeberlein M, Andalis AA (2002) Calorie restriction extends Saccharomyces cerevisiae lifespan by increasing respiration. Nature 418(6895): 344-348.

49. Howitz KT, Bitterman KJ, Cohen HY (2003) Small molecule activators of sirtuins extend Saccharomyces cerevisiae lifespan. Nature 425(6954): 191-196.

50. Anderson RM, Bitterman KJ, Wood JG (2003) Nicotinamide and PNC1 govern lifespan extension by calorie restriction in Saccharomyces cerevisiae. Nature 423(6936): 181-185.

51. Lin SJ, Ford E, Haigis M (2004) Calorie restriction extends yeast life span by lowering the level of NADH. Genes Dev 18(1): 12-16.

52. Kaeberlein M, Kirkland KT, Fields S, Kennedy BK (2004) Sir2independent life span extension by calorie restriction in yeast. PLoS Biol 2(9): E296.

53. Clancy DJ, Gems D, Harshman LG (2001) Extension of life-span by loss of CHICO, a Drosophila insulin receptor substrate protein. Science 292(5514): 104-106.

54. Wood JG, Rogina B, Lavu S (2004) Sirtuin activators mimic caloric restriction and delay ageing in metazoans. Nature 430(7000): 686-689.

55. Rogina B, Helfand SL (2004) Sir2 mediates longevity in the fly through a pathway related to calorie restriction. Proc Natl Acad Sci USA 101(45): 15998-16003.

56. Kenyon C (2005) The plasticity of aging: Insights from long-lived mutants. Cell 120(4): 449-460

57. Bartke A. Wright CJ, Mattison JA (2001) Extending the lifespan of longlived mice. Nature 414(6862): 412

58. Blüher M, Kahn BB, Kahn C (2003) Extended longevity in mice lacking the insulin receptor in adipose tissue. Science 299(5606): 572-574.

59. Chiu CH, Lin WD, Huang SY, Lee YH (2004) Effect of a C/EBP gene replacement on mitochondrial biogenesis in fat cells. Genes Dev 18(16): 1970-1975.

60. Speakman JR, Talbot DA, Selman C (2004) Uncoupled and surviving individual mice with high metabolism have greater mitochondrial uncoupling and live longer. Aging Cell 3(3): 87-95.

61. Sohal RS, Weindruch R (1996) Oxidative stress, caloric restriction, and aging. Science 273(5271): 59-63. 
62. Ristow M, Zarse K (2010) How increased oxidative stress promotes longevity and metabolic health: the concept of mitochondrial hormesis (mitohormesis). Experimental Gerontology 45(6): 410-418.

63. Langley E, Pearson M, Faretta M (2002) Human SIR2 deacetylates p53 and antagonizes PML/p53-induced cellular senescence. EMBO J 21(10): 2383-2396.

64. Luo J, Nikolaev AY, Imai S (2001) Negative control of p53 by Sir $2 \alpha$ promotes cell survival under stress. Cell 107(2): 137-148.

65. Motta MC, Divecha N, Lemieux M (2004) Mammalian SIRT1 represses forkhead transcription factors. Cell 116(4): 551-563.

66. Cohen HY, Miller C, Bitterman KJ (2004) Calorie restriction promotes mammalian cell survival by inducing the SIRT1 deacetylase. Science 305(5682): 390-392.

67. Wang Y (2014) Molecular links between caloric restriction and sir2/ sirt1 activation. Diabetes Metabolism Journal 38(5): 321-329.

68. Bertrand HA, Lynd FT, Masaro E, Yu BP (1980) Changes in adipose mass and cellularity through the adult life of rats fed ad libitum or on a life prolonging restricted diet. J Gerontol 35(6) 827-835.

69. Kershaw EE, Flier JS (2004) Adipose tissue as an endocrine organ. J Clin Endocrinol Metab 89(6): 2548-2556.

70. Barzilai N, Gabriely I (2001) The role of fat depletion in the biological benefits of caloric restriction. J Nutr 131(3): 903S-906S

71. Combs TP, Berg AH, Rajala MW (2003) Sexual differentiation, pregnancy, calorie restriction, and aging affect the adipocyte-specific secretory protein adiponectin. Diabetes 52(2): 268-276.

72. Pajvani UB, Scherer PE (2003) Adiponectin: systemic contributor to insulin sensitivity. Curr Diab Rep 3(3): 207-13.

73. Picard F, Kurtev M, Chung N (2004) Sirt1 promotes fat mobilization in white adipocytes by repressing PPAR $\gamma$. Nature 429(6993): 771-776.

74. Cohen HY, Miller C, Bitterman KJ, Wall NR, Hekking B, et al. (2004) Calorie restriction promotes mammalian cell survival by inducing the SIRT1 deacetylase. Science 305(5682): 390-392.

75. Lee CH, Evans RM (2002) Peroxisome proliferator-activated receptor- $\gamma$ in macrophages lipid homeostasis. Trends Endocrinol Metab 13(8): 331-335.

76. Mattson MP (2003) Gene-diet interactions in brain aging and neurodegenerative disorders. Ann Intern Med 139(5): 441-444.

77. Mattson MP, Duan W, Lee J, Guo Z (2001) Suppression of brain aging and neurodegenerative disorders by dietary restriction and environmental enrichment: molecular mechanisms. Mech Ageing Dev 122(7): 757-778.
78. Araki T, Sasaki Y, Milbrandt J (2004) Increased nuclear NAD biosynthesis and SIRT1 activation prevent axonal degeneration. Science 305(5686): 1010-1013.

79. Omodei D, Fontana L (2011) Calorie restriction and prevention of ageassociated chronic disease. FEBS Lett 585(11): 1537-542.

80. Ghosh HS (2008) The anti-aging, metabolism potential of SIRT1. Curr Opin Investig Drugs 9(10): 1095-1102.

81. Chaudhary N, Pfluger PT (2009) Metabolic benefits from Sirt1 and Sirt 1 activators. Current opinion in clinical nutrition and metabolic care 12(4): 431-437.

82. Kensler TW, Wakabayashi N, Biswal S (2007) Cell survival responses to environmental stresses via the Keap1-Nrf2-ARE pathway. Annu Rev Pharmacol Toxicol 47: 89-116.

83. Everitt AV, Heilbronn LK, Le Couteur DG Ch (2010) Food Intake, Life Style, Aging and Human Longevity. In: Everitt AV, et al. (Eds.), Springer New York, USA.

84. Schäfer D (2005) Aging, longevity, and diet: Historical remarks on calorie intake reduction. Gerontology 51(2): 126-130.

85. Mattison JA, Colman RJ, Beasley TM (2017) Caloric restriction improves health and survival of rhesus monkeys. Nature Communications 8: 14063.

86. Cerletti M, Jang YC, Finley LW (2012) Short-term calorie restriction enhances skeletal muscle stem cell function. Cell Stem Cell 10(5): 515519.

87. Vitousek KM, Manke FP, Gray JA, Vitousek MN (2004) Caloric restriction for longevity: II-the systematic neglect of behavioural and psychological outcomes in animal research. European Eating Disorders Review 12(6): 338-360.

88. Marzetti E, Wohlgemuth SE, Anton SD (2012) Cellular mechanisms of cardioprotection by calorie restriction: state of the science and future perspectives. Clin Geriatr Med 25(4): 715-732.

89. Weiss EP, Fontana L (2011) Caloric restriction: Powerful protection for the aging heart and vasculature. Am J Physiol Heart Circ Physiol 301(4): H1205-H1219.

90. Kalm LM, Semba RD (2005) They starved so that others be better fed: remembering ancel keys and the minnesota experiment. J Nutr 135(6): 1347-1352.

91. Libert S, Guarente L (2013) Metabolic and neuropsychiatric effects of calorie restriction and sirtuins. Annual Review of Physiology 75: 669684. 